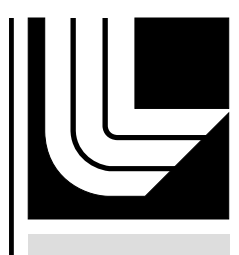

LAW RENCE LIVERMORE N A TIO N A L LABORATORY

\title{
UCRL-TR-234860
}

\section{LLNL Facility Screening Report (SCR) for B362}

G.A. Cooper

September 24, 2007 
This document was prepared as an account of work sponsored by an agency of the United States Government. Neither the United States Government nor the University of California nor any of their employees, makes any warranty, express or implied, or assumes any legal liability or responsibility for the accuracy, completeness, or usefulness of any information, apparatus, product, or process disclosed, or represents that its use would not infringe privately owned rights. Reference herein to any specific commercial product, process, or service by trade name, trademark, manufacturer, or otherwise, does not necessarily constitute or imply its endorsement, recommendation, or favoring by the United States Government or the University of California. The views and opinions of authors expressed herein do not necessarily state or reflect those of the United States Government or the University of California, and shall not be used for advertising or product endorsement purposes.

This work was performed under the auspices of the U.S. Department of Energy by University of California, Lawrence Livermore National Laboratory under Contract W-7405-Eng-48. 


\section{LLNL Facility Screening Report (SCR) for B-362}

Facility Name

Lead Preparer: Greg Cooper

Date Performed: $8 / 28 / 2007$

\section{Facility Description}

Briefly describe facility use, physical structure, location and attach a building layout: Building 362 (3,749 gross square feet) is a single-story concrete block structure with a built-up roof over steel joists. A boiler/chiller/electrical room is located in the West center portion of the building. B-362 is located near the center of the Laboratory at the corner of West Inner Loop Road and Fifth Street and is approximately 859 meters (2,818 feet) from the nearest site boundary.

B-362 is houses laboratories supporting biological activities including the use of radiological tracers.

\section{Define facility type:}

Check:

$\bigotimes \quad$ Single Structure or Area: (B/Tr/A) B-362

Complex of Buildings: Designation

Segment* of Bldg or Complex:

Seg.\#

*Attach justification for segmentation

Final Facility Classification: (Check)

$\bigotimes$ LSI $\square$ Low $\square$ Moderate $\square$ High $\square$ Nuclear Facility $\square$ Accelerator

\section{Concurrence Signatyfres for Facility Classified as LSI**:}

Lead Preparer: 14 Date: 8/30/07

AB Section Leader or designee: CM. Mn Wamendam Date: $8 / 30 / 07$

ES\&H Team Leader or designee: $M$. When D D D 8 . $8 / 30 / 07$

\section{Approval Signature for Facility Classified as LSI**: \\ Facility Management: \\ Date: $8 / 30 / 07$}

\section{Supporting Documentation Appended}

Check as appropriate:

Justification for Segmentation

Chemical Hazard List

Radiological Hazard List

Explosive Hazard List

Building Layout

** Signatures are not required on this form for facilities classified

as Low, Moderate or High. Approval signatures for these are

on the cover of the Tier 2 or

Tier 3 SBDs. 


\section{Identification of Operations, Inventories, and Hazards}

\section{List key operations that are conducted within the facility:}

Research involving analytical chemistry and microbiology.

Work with small amounts of radioactive tracer elements (C-14 and tritium)

\section{Nearby Facility threats?}

Did Facility Management receive any notifications of credible external threats from nearby LLNL facilities (>TEEL2 after mitigation)? yes $\square$ no $\square$

If yes, list the following for each notification:

\begin{tabular}{|l|l|l|}
\hline Source Facility: & Facility Contact(s): & Phone \# (s): \\
\hline
\end{tabular}

Describe hazard(s) associated with nearby facility:

\section{Hazard Identification Table}

Check the hazard types found in the facility.

\section{Not} Authorized

Authorized

$\square$

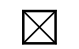

Biological Hazards

Complete block I, below

$\square$

$\bigotimes$

Chemical Hazards

Complete block II, below

$\bigotimes$

Explosive Hazards

Complete block III, below

$\bigotimes$

Radiological Hazards

Complete block IV, below

$\bigotimes$

Industrial Hazards

Complete block V, below 


\begin{tabular}{|c|c|}
\hline 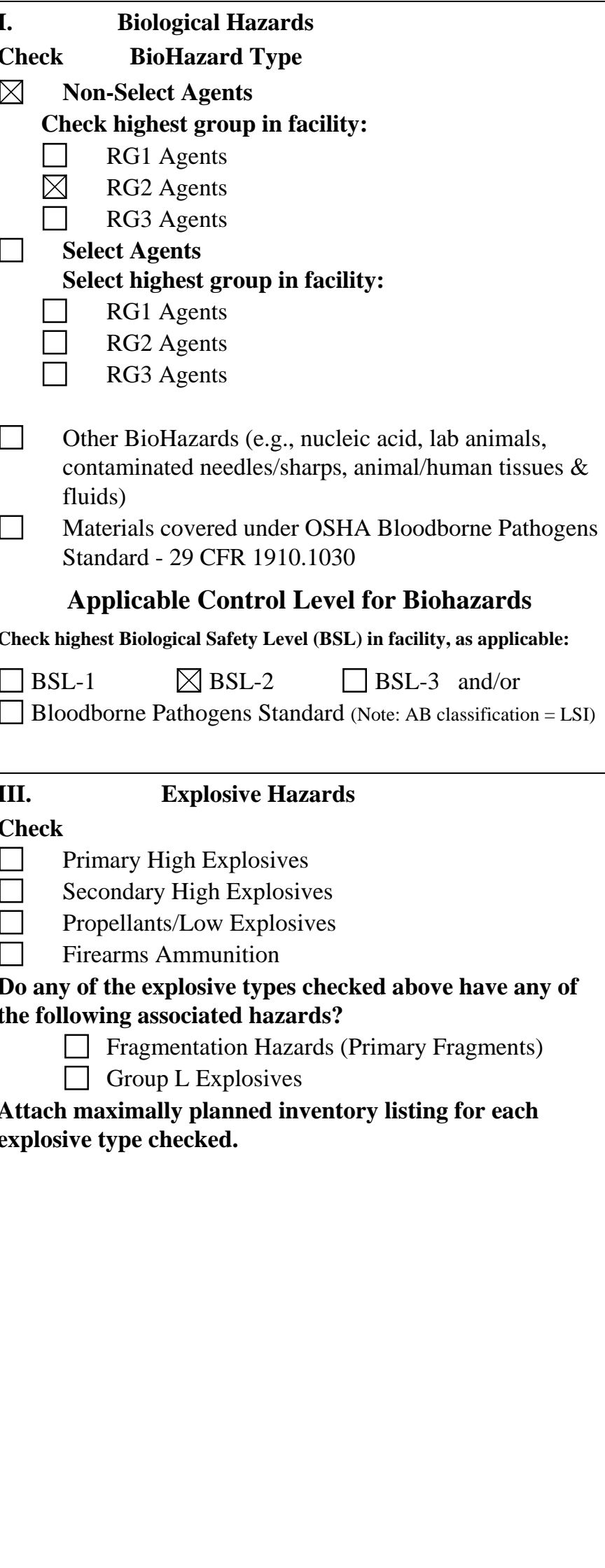 & 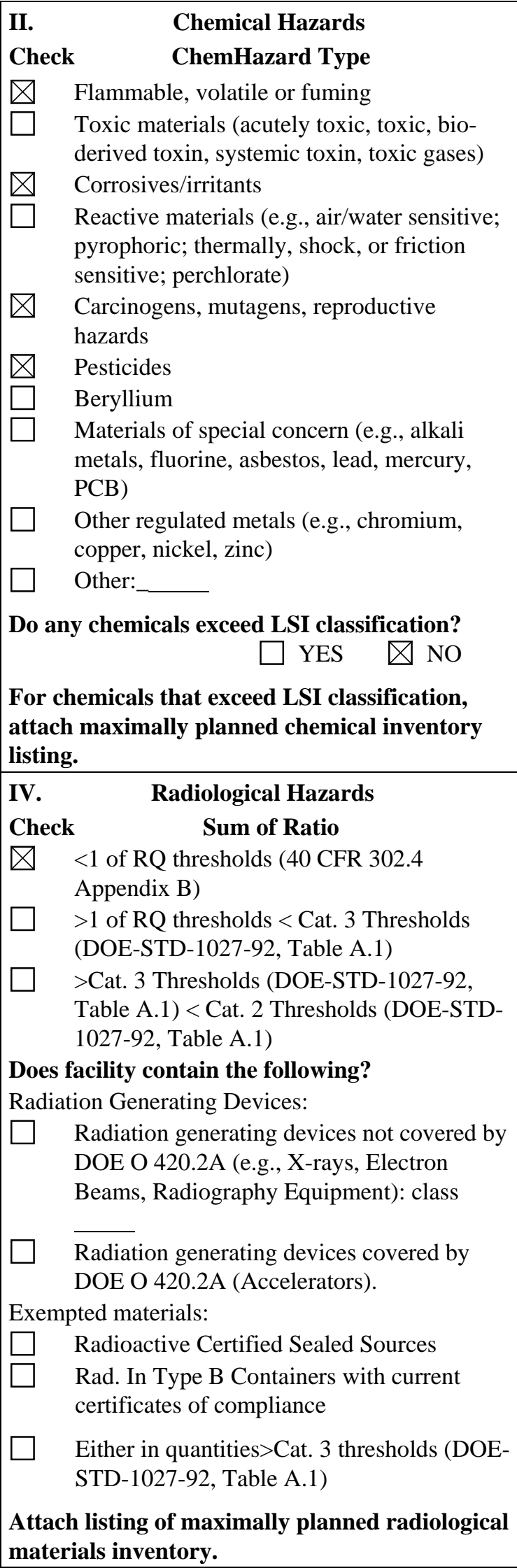 \\
\hline
\end{tabular}




\begin{tabular}{|c|c|c|c|}
\hline \multicolumn{4}{|c|}{ V. Industrial Hazards } \\
\hline $\begin{array}{l}\text { Check if } \\
\text { hazard } \\
\text { present }\end{array}$ & $\begin{array}{l}\text { Industrial } \\
\text { Hazard }\end{array}$ & $\begin{array}{l}\text { Examples of industrial hazard(s) for each general } \\
\text { category. (Select Industrial Hazards found.) }\end{array}$ & $\begin{array}{l}\text { List industrial } \\
\text { hazard(s) that } \\
\text { could directly } \\
\text { impact the public } \\
\text { (fence-line) or } \\
\text { colocated worker } \\
\quad(100 \mathrm{~m}) .\end{array}$ \\
\hline$\bigotimes$ & Electrical & 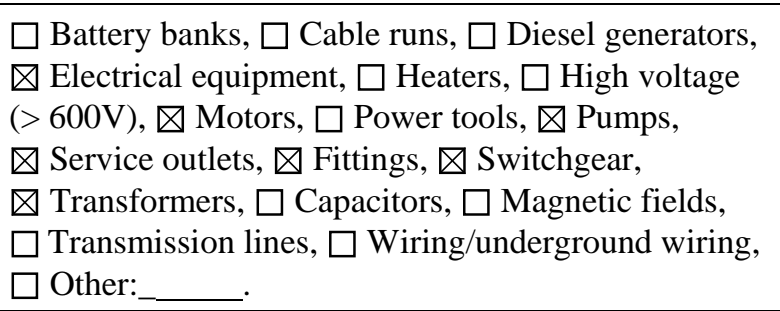 & None \\
\hline 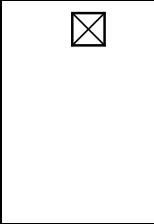 & Thermal & $\begin{array}{l}\otimes \text { Boilers, } \otimes \text { Bunsen burner/hot plates, } \otimes \text { Electrical } \\
\text { equipment, } \otimes \text { Electrical wiring, } \square \text { Engine exhaust, } \\
\square \text { Furnaces, } \square \text { Heaters, } \square \text { Lasers, } \square \text { Steam lines, } \\
\square \text { Welding surfaces, } \square \text { Welding torch, } \\
\square \text { other:__ }\end{array}$ & None \\
\hline 凶 & Kinetic & $\begin{array}{l}\square \text { Acceleration/deceleration, } \otimes \text { Bearings, } \otimes \text { Belts, } \\
\otimes \text { Carts/dollies, } \otimes \text { Centrifuges, } \square \text { Crane loads (in } \\
\text { motion), } \otimes \text { Drills, } \nabla \text { Fans, } \square \text { Firearm discharge, } \\
\square \text { Fork lifts, } \square \text { Gears, } \square \text { Grinders, } \otimes \text { Motors, } \\
\square \text { Power tools, } \square \text { Presses/shears, } \square \text { Saws, } \\
\square \text { Vehicles, } \square \text { Airplane, } \square \text { Vibration, } \square \text { Other: }\end{array}$ & None \\
\hline 冈 & Potential (pressure) & $\begin{array}{l}\square \text { Autoclaves, } \otimes \text { Boilers, } \square \text { Coiled springs, } \\
\square \text { Furnaces, } \nabla \text { Gas bottles, } \square \text { Gas receivers, } \\
\otimes \text { Pressure vessels, } \otimes \text { Vacuum vessels, } \\
\otimes \text { Pressurized system (e.g., air), } \square \text { Steam header } \\
\text { and lines, } \square \text { Stressed members, } \square \text { Other: }\end{array}$ & None \\
\hline$\bigotimes$ & $\begin{array}{l}\text { Potential } \\
\text { (height/mass) }\end{array}$ & $\begin{array}{l}\square \text { Cranes/hoists, } \square \text { Elevated doors, } \square \text { Elevated work } \\
\text { surfaces, } \square \text { Elevators, } \square \text { Lifts, } \square \text { Loading docks, } \\
\square \text { Mezzanines, } \square \text { Floor pits, } \otimes \text { Scaffolds and ladders, } \\
\square \text { Stacked material, } \square \text { Stairs, } \\
\square \text { Other:_ }\end{array}$ & None \\
\hline 凶 & $\begin{array}{l}\text { Internal Flooding } \\
\text { Sources }\end{array}$ & $\begin{array}{l}\otimes \text { Domestic water, } \square \text { Fire suppression piping, } \\
\otimes \text { Process water, } \square \text { Other: }\end{array}$ & None \\
\hline \multicolumn{4}{|c|}{ Hazard Classification } \\
\hline \multicolumn{4}{|c|}{ Select the appropriate hazard level from the dropdown menu: } \\
\hline \multicolumn{2}{|c|}{ Biological } & \multicolumn{2}{|l|}{ LSI } \\
\hline \multicolumn{2}{|c|}{ Chemical } & \multicolumn{2}{|l|}{ LSI } \\
\hline \multicolumn{2}{|c|}{ Explosive } & \multicolumn{2}{|l|}{ Not authorized } \\
\hline \multicolumn{2}{|c|}{ Radiological materials } & \multicolumn{2}{|l|}{ LSI } \\
\hline \multicolumn{2}{|c|}{ Radiation generators } & \multicolumn{2}{|l|}{ Not authorized } \\
\hline \multicolumn{2}{|c|}{ Industrial } & \multicolumn{2}{|l|}{ LSI } \\
\hline
\end{tabular}


Controls for LSI Hazards: (Controls for Low, Moderate and High hazards are addressed in Tier 2 or Tier 3 SBDs.)

\section{Briefly describe controls developed to assure that facility operations do not exceed the facility classification:}

1. Biological operations will be limited to those that can be performed at BSL-2 controls or less. All biological activities shall be reviewed via the IWS process and the LBOC prior to starting.

2. Inventories of individual chemicals shall be maintained below the lesser of the Q-values $\mathrm{Q} 1$ at 100 meters or Q0 at 600 meters.

3. CMLS manages its programmatic inventory of radiological materials to maintain and comply with a Facility Safety Basis Envelope (SBE) of LSI for B362. A Radioactive Materials Inventory System is maintained by facility management. It is reconciled as frequently as necessary to ensure that the facility radiological inventory remains below the Final RQ limits in 40 CFR 302.4 Appendix B on a cumulative sum-of-the-ratios basis for all isotopes. Inventory reconciliation more frequent than annual shall be performed if the inventory exceeds an administrative control level of $75 \%$ of the RQ limits. Prior to receipt, additions are verified not to cause the radiological inventory to exceed the RQ limits. Additions not fully characterized are estimated using field measurements and owner knowledge.

4. Industrial hazards are managed by facility management at the LSI level.

\section{Other controls?}

Briefly describe:

None

\section{List what document(s) through which the controls will be implemented:}

CMLS-332, "Chemical Management Plan”.

CMLS-406, "Radioactive Materials Inventory Management Plan”.

Individual Integration Worksheets (IWSs). 


\section{B362 RADIOLOGICAL INVENTORY}

\begin{tabular}{|c|c|c|c|c|c|c|c|c|c|c|c|}
\hline Nuclide & $\begin{array}{c}\text { Initial } \\
\text { Quantity } \\
\text { [activity or } \\
\text { mass] }\end{array}$ & Units & $\begin{array}{c}\text { Initial } \\
\text { Activity } \\
\text { [Ci] }\end{array}$ & $\begin{array}{c}\text { Reference } \\
\text { Date }\end{array}$ & $\begin{array}{c}\text { Specific } \\
\text { Activity } \\
{[\text { Ci/g] }}\end{array}$ & $\begin{array}{c}\text { Halflife } \\
\text { [sec] }\end{array}$ & $\begin{array}{c}\text { Decay } \\
\text { Corrected } \\
\text { Activity [Ci] }\end{array}$ & $\begin{array}{l}\text { Final } \\
\text { RQ } \\
\text { [Ci] }\end{array}$ & $\begin{array}{c}\mathrm{RQ} \\
\text { Fraction }\end{array}$ & $\begin{array}{c}\text { Cat III } \\
\text { Threshold } \\
\text { [Ci] }\end{array}$ & $\begin{array}{c}\text { Cat III } \\
\text { Fraction }\end{array}$ \\
\hline C-14 & $1.86 \mathrm{E}+04$ & uCi & $1.86 \mathrm{E}-02$ & $04 / 11 / 07$ & $4.46 \mathrm{E}+00$ & $1.81 \mathrm{E}+11$ & 1.86E-02 & 10 & 1.86E-03 & $4.20 \mathrm{E}+02$ & 4.44E-05 \\
\hline $\mathrm{H}-3$ & $1.17 \mathrm{E}+04$ & uCi & 1.17E-02 & $04 / 11 / 07$ & $9.65 E+03$ & $3.90 \mathrm{E}+08$ & 1.15E-02 & 100 & 1.15E-04 & $1.00 \mathrm{E}+03$ & 1.15E-05 \\
\hline I-129 & $1.00 \mathrm{E}+00$ & uCi & 1.00E-06 & $04 / 11 / 07$ & 1.77E-04 & $4.95 \mathrm{E}+14$ & $1.00 \mathrm{E}-06$ & 0.001 & 1.00E-03 & 6.00E-02 & 1.67E-05 \\
\hline
\end{tabular}

\begin{tabular}{lccc} 
Nuclide & Decay Corrected Activity [Ci] & RQ Fraction & $\begin{array}{c}\text { Cat III } \\
\text { Fraction }\end{array}$ \\
\hline C-14 & $1.86 \mathrm{E}-02$ & $1.86 \mathrm{E}-03$ & $4.44 \mathrm{E}-05$ \\
H-3 & $1.15 \mathrm{E}-02$ & $1.15 \mathrm{E}-04$ & $1.15 \mathrm{E}-05$ \\
I-129 & $1.00 \mathrm{E}-06$ & $1.00 \mathrm{E}-03$ & $1.67 \mathrm{E}-05$ \\
Grand & $3.01 \mathrm{E}-02$ & & \\
Total & & $2.98 \mathrm{E}-03$ & $7.25 \mathrm{E}-05$ \\
\hline
\end{tabular}

Inventory table represents the typical types and quantities of nuclides used within this facility, the exact types and quantities may change somewhat over time, however, total nuclide inventory will be maintained within LSI classification limits 


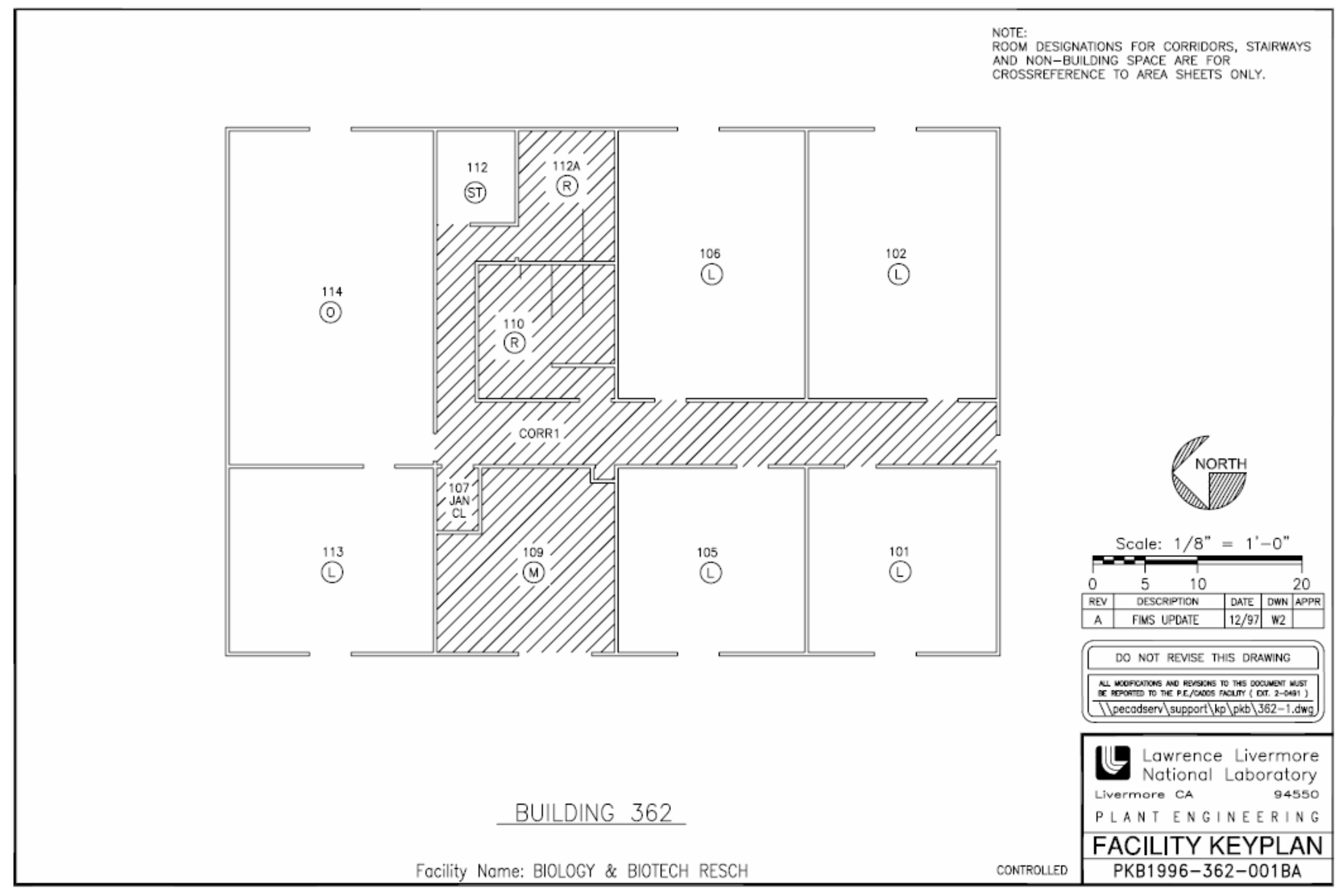

